\title{
A Multiple Sensor-based Shoe-mounted User Interface Designed for Navigation Systems for the Visually Impaired
}

\author{
J. ZHANG, C.W. LIP, S.K. ONG, A.Y.C. NEE \\ Mechanical Engineering Department \\ National University of Singapore \\ Singapore \\ \{mpezhanj, u0508779, mpeongsk, mpeneeyc\}@nus.edu.sg
}

\begin{abstract}
Assistive technology and assistive devices have been researched and developed to promote an independent daily life for visually impaired individuals. Navigation for the visually impaired may be one of the most challenging tasks due to high expectations on both the functionality and the mechanical specifications of the devices. This research aims at developing a multiple sensor-based hand-free device as a navigation aid to detect obstacles. The developed device incorporates a sensor unit and a feedback unit with either a wired or a wireless data communication setup. The working principle and the design criteria are identified with several working scenarios for potential applications. The implementation of the design into a prototype device is discussed in details. The prototype has been tested, and recommendations of possible improvements and future works are proposed.
\end{abstract}

Keywords-navigation system for visually impaired; shoemounted asssitive device; infrared-based proximity detection

\section{INTRODUCTION}

Assistive technology (AT), aiming at providing assistance to disabled people to improve their quality of life, has been experiencing immense development over the recent years. In particular, AT technology and devices have been researched and developed for the visually impaired individuals [1] to enhance their mobility, communication ability and promote their self-esteem in the society. Suffering inconveniences caused by their visual loss or blindness, difficulties in numerous aspects of daily living have been encountered by the visually impaired individuals. Among all the difficulties, solving the navigation problem may be one of the most challenging because of the high expectations on both the functionality and the mechanical specifications that are imposed on the design of such navigation aids. Navigation Systems for the Visually Impaired (NSVI) have to meet several criteria. Firstly, the system must be able to provide reliable guiding information during the course of navigation, either in indoor or outdoor settings. Secondly, the system should be small, easy to handle, and light in weight so that it will not be an extra burden for the user to carry along the trip. Thirdly, the system should be as inconspicuous or non-intrusive as possible as the visually impaired individuals usually would not want to be stared at by others. For these reasons, while developing NSVI for the visually impaired, researchers tend to develop small devices that can be attached to the apparatus normally used by the visually impaired (such as the white cane), to the user's body, or to the apparel and shoes.

Navigation systems are targeted at addressing one or more typical navigation problems encountered by the visually impaired, e.g., way-finding, obstacle avoiding, crossing roads, etc. Specifically, the user's position and orientation must be recognized in real time; obstacles and traffic signs (traffic lights, zebra crossings, etc.) must be identified. Different technologies have been incorporated into the development of navigation aids based on positioning systems and computer vision $(\mathrm{CV})$ technology. In addition, body movement analysis plays an important role in pedestrian navigation. During walking, the movement of the feet provides better information and properties for scientific analysis, such as the higher accelerations generated by the stride, and the highly repeated stride cycle with a stance phase and a swing phase alternatively [2]. Hence, research on developing shoe-mounted devices using inertial sensors and, more recently, low cost cameras have been employed for obstacles and context detection purposes. Furthermore, sensory replacement is also essential in that the instructional messages from the navigation aid must be conveyed to the user effectively. Audio messages or tactile feedbacks are the two approaches frequently exploited by researchers [3].

This research focuses on the development of a multiple sensor-based user interface for the visually impaired as a supplementary device to the white cane, which is limited to detecting obstacles within two feet ahead of the user. Within such a short range, it is difficult for the visually impaired to circumvent the potential danger with the aid of a white cane. The objective for this research is to facilitate the detection of obstacles or dangers far ahead of the user taking into consideration the reflectance of the road surface to facilitate obstacle detection. The user interface comprises of a sensor unit and a feedback unit. For road surface reflectance measurement, the sensor unit is shoe-mounted, applying infrared (IR) technology. Incorporating multiple sensors, the detection will be realized by both direct distance detection and 
road surface reflectance detection. According to the research by Ohtsuka et al. [4], the arms and the back of the visually impaired are usually more sensitive to tactile stimulations, and thus the feedback unit in this research is designed to be armattached. Regarding the information rendering approaches, constant tactile feedback is provided in an attempt to provide the user with a higher level of perception of the surroundings, and hence a stronger confidence during environment exploration. Both wired and wireless configurations have been investigated for the device. It has been found that the device will indeed introduce minimum hindrance to the walking behavior when incorporating a wireless data communication technology.

The rest of this paper is organized as follows. Section 2 discusses in details the state of the art in the developed technologies used in NSVI. Shoe-mounted devices for navigation purpose are discussed separately in this section. Section 3 presents the design principles and criteria considered during the development of the multiple sensor-based shoe. Development of a prototype product will be presented in Section 4, together with a discussion on the implementation details. Section 5 presents the potentials of the product and some possible future works.

\section{LITERATURE REVIEW}

\section{A. NSVI}

According to the navigation methodologies employed in NSVI research, the navigation systems can be categorized into two groups, i.e., positioning system-based and CV-based.

Two types of system settings have been utilized in the literature on NSVI, viz., global positioning system (GPS) and local positioning system. Emerging in the early 70's of the last century, GPS has evolved from exclusive military applications to everyday usage. Most newly launched cell phones have been embedded with GPS receivers. Integrated with a GPS receiver, a NSVI system can give navigation instructions with respect to a given map. However, GPS is only applicable in outdoor navigation, and the accuracy is greatly hindered by bad climate, close buildings, etc. On the other hand, local positioning systems exploit the pilot-and-beacon technology, inertial sensors and dead reckoning (DR) technology, etc., to detect the relative movements of the users, such as the moving speed, the heading direction, etc. A NSVI system was reported by Cecelja et al. [5], where they utilized GPS and Geographic Information System (GIS) for absolute global positioning and navigation. At the same time, a DR system was employed as an alternate when the GPS service is not available. A camera was integrated in their prototype system. However, no image processing or $\mathrm{CV}$ technique was employed. Images captured by the camera were transmitted to a human guide via a mobile network. Inspecting the camera images and the environment map, the guide provides verbal orientation instructions to the individual with visual impairment. It is no doubt that a human guide can assist the navigation more promptly, but the efficiency of human guidance is questionable. Ran et al. [6] developed Drishti, an NSVI utilizing GPS and ultrasonic pilots for outdoor and indoor navigation, respectively. Pilots were installed in the corners of a room, while beacons were attached on the user to receive ultrasonic signals. Ross and Blasch [7] employed an IR receiver and a virtual sonic beacon in outdoor navigation. Components in off-the-shelf television IR remote control systems were utilized. The research focused on improving the responding range to be up to 100 feet, and thus outdoor applications can be accomplished.

RFID (Radio Frequency Identification) technologies have been widely applied nowadays for object identification, asset or personnel tracking and positioning, etc. Pre-installed RFID tags containing contextual information (such as surrounding landmarks, turning points, etc.) can be applied in NSVI. For example, RFID readers may be attached at the end of a white cane. When a user scans the ground with the white cane, the system reads in the information and synthesizes instructions accordingly. In this case, short-range RFID readers are normally applied as they are small and light to carry. Shiizu et al. [8] combined RFID tags with color sensors for an indoor navigation application. RFID tags were installed along color lines, which were painted on the ground and declared to be widely employed in Japan. As the user is directed by the color sensor to follow a certain line, he does not have to scan the floor actively, hence eliminating any effort to search for tags. However, directional information stored in these RFID tags is restricted to be path-oriented. For example, a turning-point tag installed on a red line indicates only the turning point of that red line. RFID tag grids were investigated as a substitution for the tactile ground surface indicator [9]. In this research, absolute directional information is provided by the pattern alteration of the tag information. Another research shows two examples of the navigation through a staircase and the end of a corridor using four and three RFID tags, respectively [10]. Absolute directional information is provided based on an analysis of the tag-detecting sequence. In the navigation of a staircase, the detection sequence disambiguates the possibilities of facing an upward or downward staircase.

It should be noted that positioning system-based NSVI focuses on identifying the position of a user rather than analyzing the environment around the user. The environment must be prepared, i.e., an environment map is required and an essential infrastructure is a must, such as the installation of pilots and beacons, RFID tags, the availability of the GPS satellites, etc. Information with respect to the surrounding buildings or objects is retrieved according to the map, based on which the current user position and heading direction are estimated. In other words, such systems cannot provide updated or dynamic context information.

In CV-based NSVI, cameras are utilized to capture the environments, especially the scene in front of the users. There are basically two research issues in CV-based NSVI, viz., depth perception and object recognition. Stereo cameras (two cameras that have been calibrated) are usually applied for depth perception, while a single camera is applied to extract features, edges, etc., so as to conduct simple object recognition, such as doors, stair steps, pedestrian crossings, etc. Zelek [11] reported a prototype NSVI for a way-finding task in both indoor and outdoor navigation. A commercial stereo camera was utilized, and a neural network-based scene recognition method was employed to determine the location of the user, i.e., either indoor or outdoor. Face detection method was also developed 
to recognize the pedestrian who is probably talking to the user. Similarly, space cognitive maps were obtained from stereo images [12] to reflect the nearest border of the obstacles in front of the user. Sainarayanan et al. [13] employed a different scenario of using stereo cameras. A fuzzy cluster-based image processing method was developed to enhance the captured stereo images by separating the background and objects more sharply. Sonification of the images was conducted to convert the stereo images to stereo sounds. For NSVI utilizing only single cameras, contextual features can be obtained from image processing, especially from edge detection. Contextual features in indoor navigation mainly include door and corridor detection. Sarfraz and Rizvi [14] proposed a navigation system using edge detection to recognize doors based on the assumption that doors usually have a different color from the wall. Lee and Leung [15] developed indoor navigation based on certain indoor visual cues. For example, two edges between a corridor and the ceiling are compared. If the edge on the right side is shorter than the one on the left, it probably means the corridor has a right turn ahead. Recognition of door handles was integrated into door detection, given that door handles have a known ' $\mathrm{L}$ ' or ' $\mathrm{T}$ ' pattern. In outdoor navigation, detection of environmental objects and determination of the user's orientation are essential. Kaluwahandi and Tadokoro [16] employed simple vertical or horizontal image projection to detect the orientation angle of pedestrian crossings and white lanes. Barcode tags are used in a similar way as the RFID tags. Iannizzotto et al. [17] employed a simple barcode pattern as a 3D marker. The motion of the camera can be estimated using $\mathrm{CV}$ technology, while at the same time the barcode can be translated. Tjan et al. [18] developed a Digital Sign System (DSS), in which barcodes are printed on retro-reflective material so that the tags can be segmented more reliably. A specific coding scheme was employed by Ebrahim et al. [19] to directly include useful information in the tags to indicate not only an ID number but also the building name, office hours, etc., in indoor and outdoor navigation.

Effectively passing the information gathered by the system to the user is another research topic in the literature of NSVI. Audio messages and tactile feedbacks are the two most frequently exploited approaches. As natural as they are, artificial voice-based verbal instructions and scene descriptions have been utilized in NSVI to assist the user to understand the surroundings [8, 16-19]. At the same time, some researchers developed image-to-sound substitution mechanisms. In vOICe [20], instead of providing descriptive audible voices, images will be scanned and transformed into tones with different pitches and lengths according to the pixel intensity levels. After training, the user will set up a correspondence between the tones and the entire image to draw a mental image. Sainarayanan et al. [13] adopted a similar conversion scheme, but image pairs from a stereo camera were converted and transmitted to a stereo headset. Image-to-tactile substitution has also been widely adopted. Stereo cameras are usually adopted and disparity maps are mapped into analog signals to actuate physical devices. The development of these physical devices is essential in these research studies, e.g., the dynamic tactile map developed by Maingreaud et al. [12]. Disparity maps from a stereo camera are sampled and mapped onto a board comprising a number of micro-coils (e.g., $8 \times 8$ in the prototype). By touching the board, the user can understand the profiles of the obstacles in front of him. In the research by Zelek [11], a tactile glove was developed to substitute the user's visual perception. Disparity maps are divided into several sections, and each of them corresponds to a motor installed in the glove. If the perceived depth in one section is small, a vibration signal will be sent to the respective motor so that the user will be alerted. Johnson and Higgins [21] developed a belt combining two webcams and 14 motors. A disparity map generated by the two cameras will be divided into vertical slices and converted to intensity signals according to the nearest depth value in each slice. These signals will actuate the motors in the belt.

\section{B. Shoe-Mounted Devices}

Most shoe-mounted devices for navigation purposes approximate the user movement with respect to the starting position, and subsequently assist the navigation according to a given environment map. Data from sensors, such as pedometer, accelerometer, gyro, etc., are processed for this purpose. Compared with approaches that exploit torso-mounted sensors to approximate a user movement, shoe-mounted (or footmounted) devices are able to provide more reliable estimation result due to the highly repeated cyclic feature of a human stride. For example, Foxlin [22] incorporates a commercial inertial sensor in a pedestrian tracking system. To solve the tilt drifting problem, the researcher applied the Zero-Velocity UPdates (ZVUPs) measurement during each of the stance phase when the foot is nearly still on the ground. A magnetic compass was applied to correct the heading drift problem. Godha and Lachapelle [23] incorporated several technologies, which include INS (inertial navigation system), GPS and ZVUPs, into a pedestrian navigation system that is applied in indoor and outdoor environments.

There has been recent research on gait monitoring, analysis and rehabilitation using body-mounted devices, specifically foot-associated devices. Chen et al. [24] presented the development of a shoe-integrated device, incorporating force, flexion and inertial sensors, to detect abnormal gait for clinical analysis purposes. Kong and Tomizuka [25] implemented air pressure sensors in a shoe for gait monitoring purposes. Liu et al. [26] incorporated several gyroscopes and accelerometers attached to the foot, ankle and knee to measure the gait. CV technology has been incorporated into shoe-mounted devices. A system using a shoe-mounted camera together with inertial sensors was developed by Fitzpatrick and Kemp [27] for gait analysis and obstacle detection purposes. The researchers made a reasonable assumption that the lower part of the camera image during the stance phase is the floor. This facilitates the floor segmentation and further image analysis afterwards.

On the other hand, researchers have explored the Walking in Place (WIP) technology for VR applications and rehabilitation purposes. The WIP technology allows a user to explore a virtual environment (VE) by physically walking in one place, detecting the user locomotion using foot- and legmounted sensors or apparatuses, such as treadmills. A series of sensor-based shoe-mounted devices have been developed [28] to facilitate the detection of foot motion, and pedestrian moving direction indicated by the foot rotation, etc. Rey et al. 
[29] exploited a commercial optical tracking system to facilitate WIP navigation in a VE, where an optical tracking system helps to determine the velocity and rotation of the user's feet.

\section{Multiple Sensor-BASEd User InTERface Design}

\section{A. Working Principle}

Inspired by the obstacle detection and line following function modules usually installed on navigation robots, the multiple sensor-based user interface was proposed to work with IR sensors, which give the device the ability to detect obstacles ahead of the user and the reflectivity of the surface the user is standing on. The device incorporates two units, sensor unit and feedback unit to be mounted on the user's body (Fig. 1a). Multiple sensors are integrated in the sensor unit, including two IR proximity sensors installed underneath the shoe and a long range distance sensor installed in front of the shoe for road surface reflectance detection and obstacle detection, respectively (Fig. 1b). IR lights, which are emitted from the emitters of these IR sensors, are reflected from a reflective surface within a specific range and detected by the IR receiver, thus generating a voltage as the output. The voltage generated can be translated into a signal indicating the presence of a reflective surface in the detecting range. The signal is then sent via a wireless RF transmission or wired data communication to the feedback unit worn by the user. This feedback unit will provide vibration signals to the user based on the obstacles and surfaces detected by the sensors.

To work as a supplement to a white cane, the detecting range of Sensor A (Fig. 1b) should be about $80-100 \mathrm{~cm}$ and above. Sensor B (Fig. 1b) is used to detect the road surface reflectance, and a range of about $10 \mathrm{~cm}$ will be sufficient. The user can differentiate a few scenarios based on the combinations of vibration signals received. These different scenarios are listed as follows:

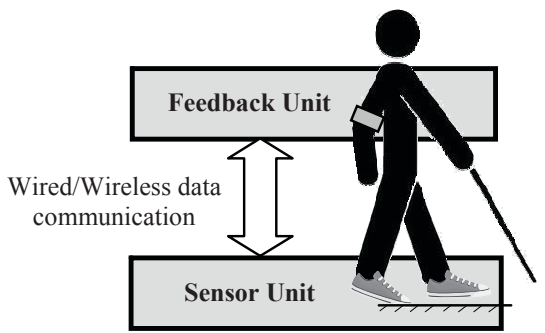

(a) The proposed user interface overview

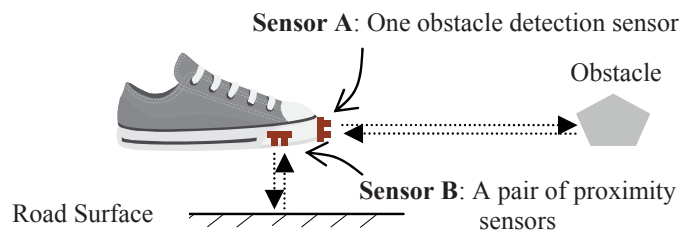

(b) Shoe-mounted sensors

Figure 1. Working principle of the multiple sensor-based shoe
Scenario 1: An obstacle is detected ahead of the user by Sensor A. This is useful for the visually impaired to prevent tripping. Vibrations from one or both vibration motor(s) will be felt by the user according to the distance from the obstacle.

Scenario 2: A black surface or a step is detected underneath the shoe of the user by Sensor $\mathbf{B}$. This is useful for the visually impaired to detect steps down a stairway or a black surface marking to indicate the existence of a danger zone ahead. Vibrations from two vibration motors will be felt by the user.

Scenario 3: With a switch to toggle the interpretation of data received by the sensors, the user can feel the ground under his shoe becoming closer. This is useful for rehabilitation purposes where a user has to keep his leg raised at a certain height. Vibrations from one or both vibration motor(s) will be felt according to the height of the user's foot from the ground.

\section{B. Device Mounting and Securing Mechanism}

A platform to house the sensor unit circuitry was designed to be worn underneath the shoes of the user. Certain properties and requirements are expected of the platform in order to provide comfortable wearing experience and optimal system performance. The material chosen for the platform must be able to withstand the weight of the user, have good wear resistance, waterproof and preferably able to offer cushioning support to the user. The materials being considered are thermoplastics, Styrofoam, rubber and rubber foam. Thermoplastics, such as ABS, are durable and light. However, thermoplastics are too rigid, which makes it uncomfortable for the users to wear for long periods of time. Styrofoam is less rigid but wears out easily, especially when in contact with water. Rubber on the other hand provides the required durability and cushioning effect while remaining waterproof. However, for the amount of rubber needed to manufacture the platform, it proves to be too heavy for the user walk around without exerting any strain on the legs. Rubber foam possesses the properties that the platform required, which is light, waterproof and durable. In addition, it is sufficiently elastic to provide cushioning to the foot while rigid enough to support the weight of the user, such that it does not deform too much when a load is applied. Rubber foam is also very affordable. Hence, rubber foam was used for the prototype of the platform.

The platform is designed to fit the base of a common shoe. An entrenchment is created in the front portion of the platform to house the sensor unit circuit board. Small slots are made at the front and side of the platform for the placement of the sensors, wires, etc. By placing the sensors at the appropriate positions, these sensors will be able to detect obstacles ahead of the platform as well as black surfaces when the user is stepping on them. The switches on the side allow the user to conveniently switch off the device when not in use in order to conserve power and to toggle the functionality of the device. An illustration of the platform design is shown in Fig. 2a.

To secure the platform onto a shoe, several criteria have been taken in consideration. Firstly, the platform should be transferable between shoes so that the user only needs to purchase one set of the device. Secondly, no permanent alterations should be made on the shoes. This is to ensure that 
the shoes remain intact and functional even when the platform is not attached to the shoes. Thirdly, it should be relatively easy to secure the platform onto the base of the shoe. A flexible step-on-and-fasten mechanism was adopted to secure the platform onto the shoe, as shown in Fig. 2b. Straps with Velcro patches are attached onto the front and back of the platform, which can thus be secured onto the shoe by strapping the Velcro over the shoe at the front and strapping around the ankle of the user at the back of the platform. This ensures an even distribution of load on the platform at both ends and the platform does not fall off. The straps can be easily strapped on and removed.

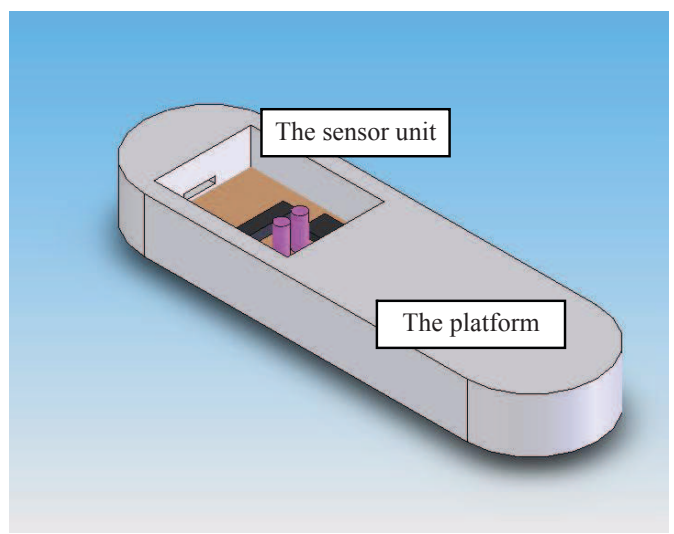

(a) Illustration of the platform with electronics embedded

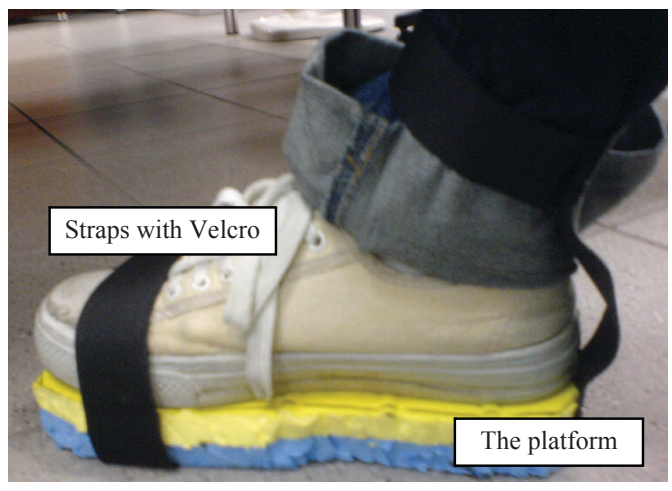

(b) Illustration of the step-on-and-fasten mechanism

Figure 2. Platform securing mechanism

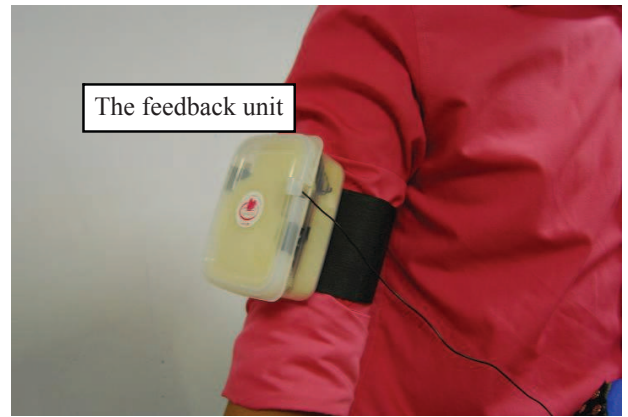

Figure 3. Illustration of securing the feedback unit onto the user's arm
Sensor Unit

Feedback Unit

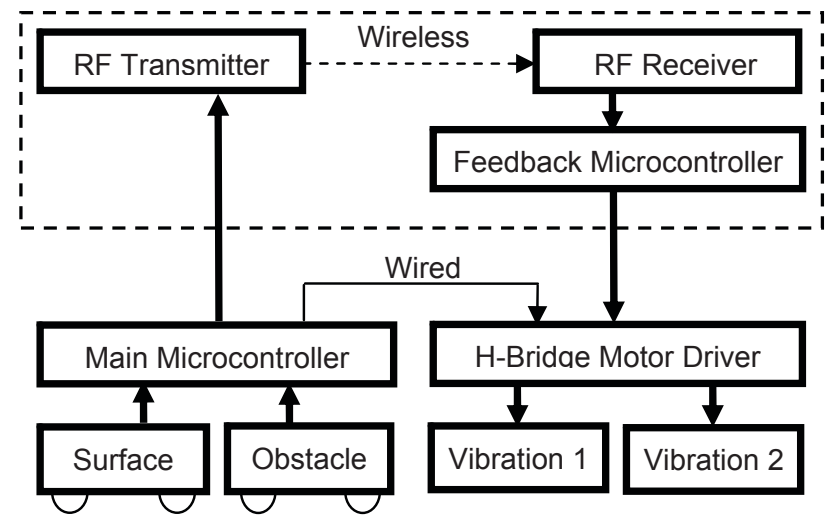

Figure 4. The system overview in function blocks in two communication configurations

On the other hand, the circuitry of the feedback unit is placed in a casing as the vibration motors have to be firmly held in order to create effective vibration feedbacks that can be felt by the users. Keeping the motors firmly in place converts all the kinetic energy produced from the rotation of the motors to vibrations throughout the casing. Minimum movement of the vibration motors also reduces the cyclic loading on their wiring, which may lead to the breakage of the wire connections due to fatigue. Fig. 3 shows a prototype of the feedback unit secured around the user's upper arm. Data communication between the sensor unit and the feedback unit can be achieved by wired communication or RF wireless communication.

\section{IMPLEMENTATION AND EXPERIMENTS}

\section{A. System Overview}

Based on the working principle presented in the earlier sections, a functional block diagram of the multiple sensorbased user interface is presented in Fig. 4, showing both the wireless and wired configurations. The device includes one microcontroller and three IR-based sensors (two proximity sensors and one obstacle detection sensor) on the sensor unit side, and two vibration motors driven by an H-bridge motor drive on the feedback side. For the wireless configuration, a pair of RF transmitter and receiver and a feedback microcontroller are needed for data communication purpose.

\section{B. Hardware Implementation}

\section{1) Microcontroller}

There are several types of commercially available microcontrollers, while the most common types are probably the AVR and PIC microcontrollers. Each microcontroller has a different environment and is coded in a different manner. In addition, the user can choose from a variety of programming languages to program the microcontroller, ranging from the low level assembly languages, where the system is more efficient but requires the users to have a stronger knowledge on microcontroller system architecture, to the higher level programming languages, such as $\mathrm{C}, \mathrm{C}++$, etc. For the prototype development, a PIC16F877A microcontroller from Microchip 
was employed. A LM7805 voltage regulation chip was used to convert a $9 \mathrm{~V}$ battery power source to $5 \mathrm{~V}$ that is required to operate the microcontroller. The programming language used for this microcontroller is $\mathrm{C}$ language.

\section{2) IR-based Proximity Sensor and Obstacle Detection} Sensor

IR-based proximity sensors that can be easily acquired from the market were considered according to the specification analysis discussed in Section 3.1. For the surface reflectance sensors (Sensor B in Fig. 1b), two medium range proximity sensors (Model IR01A from Cytron Technologies) were used, as shown in Fig. 5a. This sensor model has an adjustable detecting range from $2 \mathrm{~cm}$ to $10 \mathrm{~cm}$. A $5 \mathrm{~V}$ power supply is needed for this sensor, and it outputs a HIGH when no obstacle is detected and a LOW when a non-black object is detected within its range. The output signals are connected to the RD2 and RD3 pins of the main microcontroller, which can then be used for comparison in the code. Fig. 5a shows this hardware implementation, which is mounted inside the entrenchment of the platform with the IR transmitter and receiver facing towards the ground. These two IR01A sensors are implemented with different detecting ranges for two reasons. Firstly, the sensor with the detecting range of $2 \mathrm{~cm}$ can report the stance phase of the foot when it is almost on the ground. Hence, the output from the obstacle detecting sensor will be read in only when this IR01A sensor output is LOW. This mechanism avoids wrong data reading when the foot is more than $2 \mathrm{~cm}$ above the ground, i.e., where the foot is probably during a stride cycle. In this case, the foot may be pointing towards the ground, and the obstacle sensor will detect the distance between the foot and the ground, and the device will send wrong alarms to the user. Secondly, the output of these two sensors (detecting range of $10 \mathrm{~cm}$ and $2 \mathrm{~cm}$, respectively) will have three combinations of output, i.e., (HIGH, HIGH), (LOW, HIGH), and (LOW, LOW), when the foot is above the ground at a distance of more than $10 \mathrm{~cm}$, between $2 \mathrm{~cm}$ and $10 \mathrm{~cm}$, and below $2 \mathrm{~cm}$. This allows for lower-limb rehabilitation exercises by judging the combinations read by the microcontroller. As a result, the patient will receive corresponding instructions or stimulations according to his foot movements.

A long-range IR-based proximity sensor (model GP2Y0A02YK from Sharp) was applied for the obstacle detection purpose, as shown in Fig. 5b. This sensor is able to detect obstacles up to a range of $150 \mathrm{~cm}$, providing an analog signal output. Based on the sensor specifications, when the sensor detects an obstacle that is more than $20 \mathrm{~cm}$ ahead, an output voltage of about $2.7 \mathrm{~V}$ will be generated, and this output voltage will keep decreasing to below $0.5 \mathrm{~V}$ when the distance between the sensor and the obstacle increases to up to $150 \mathrm{~cm}$. The output of the sensor is connected to the RA0/AN0 pin for analog-to-digital conversion. Two threshold values were defined in the codes so that the system can report obstacles detected within a dangerous distance of $80-120 \mathrm{~cm}$ or within a less dangerous distance of $120-150 \mathrm{~cm}$. In this way, the system can report obstacles that cannot be detected by the white cane within that two particular distance ranges by vibrating one or two of the vibration motors, and the user will be more prepared for the changes along the way. This sensor is powered by the same battery after being regulated to $5 \mathrm{~V}$ by the LM7805.

\section{3) Feedback unit}

Two mini $3 \mathrm{~V}$ vibration motors (as shown in Fig. 6) are applied to provide feedback to the user. However, as the vibration motor has a maximum operating voltage of $3.5 \mathrm{~V}$ and requires at least $1 \mathrm{~A}$ of current to function, it cannot be driven directly by the microcontroller. Hence, a L293D H-bridge IC module was used to drive the vibration motors. A separate power supply of $3 \mathrm{~V}$ was also provided as the power supply for the vibration motors. The H-bridge acts as a bridge between the $3 \mathrm{~V}$ power supply and the vibration motors while taking TTL signals from the microcontroller. When the microcontroller sends a HIGH signal to the H-bridge enable pin, the H-bridge allows the current to flow from the $3 \mathrm{~V}$ power supply to the vibration motor to allow it to function. Similarly, when the microcontroller sends a LOW signal to the enable pin, the Hbridge module breaks the current flow from the $3 \mathrm{~V}$ power supply to the vibration motor, causing it to stop functioning. The L293D H-bridge module has two enable pins and is able to drive two vibration motors separately, and this fits the requirement of the proposed implementation scenarios. The circuit of the feedback unit based on the wired communication configuration is shown in Fig. 6. Table 1 shows the configurations of the two vibration motors when different scenarios are considered.

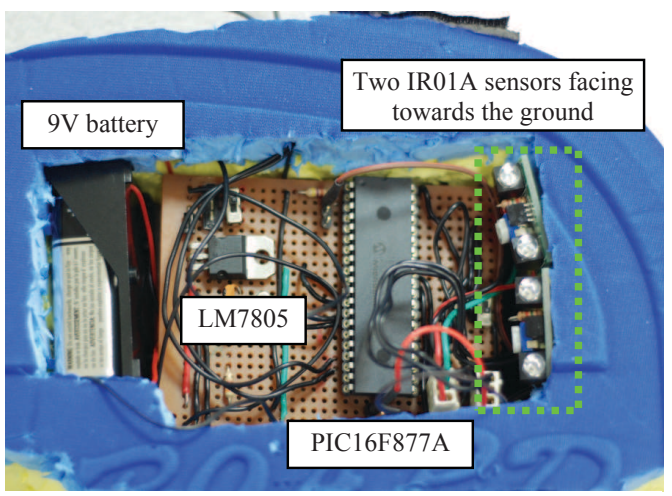

(a) Bottom view of the platform

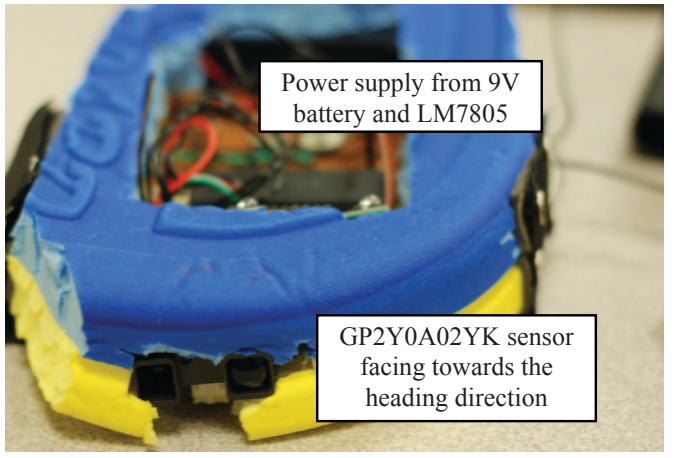

(b) Front view of the platform

Figure 5. Implementation of the IR proximity sensors

\section{Data Communications}

Both wired and wireless connection between the microcontroller of the sensor unit and the microcontroller of the feedback unit can be established. For the wired 
configuration, as there are only two sensors, two output pins are required to relay the data from the microcontroller of the sensor unit to the enable pins of the H-bridge in the feedback unit. For the wireless configuration, the microcontroller in the feedback unit will send signals to the H-bridge upon successful data communication between the RF modules. The H-bridge can thus drive the correct vibration motors, in turn signaling the types of scenarios to the user. The vibration motors will be driven according to the pre-defined scenarios described in Section 3.1.

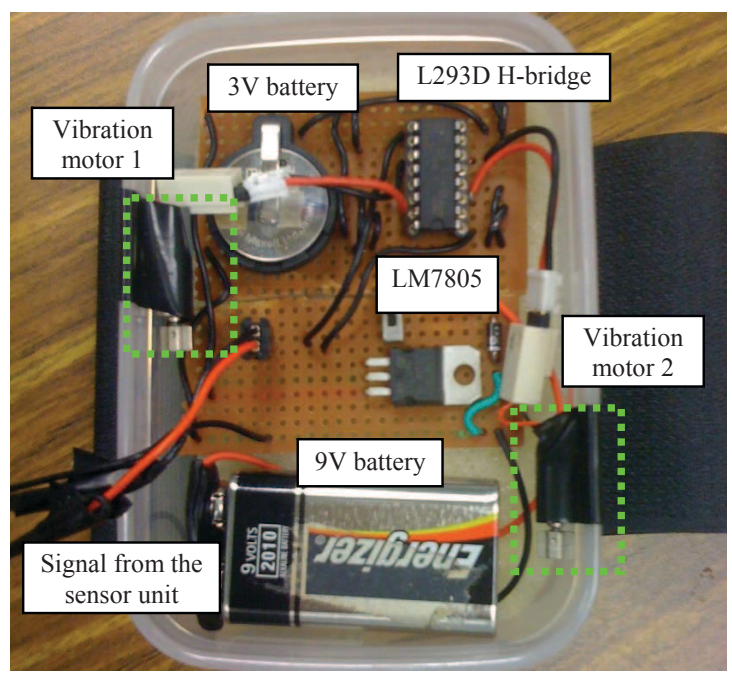

Figure 6. Implementation of the feedback unit in wired configuration

TABLE I. CONFIGURATIONS OF THE TWO VIBRATION MOTORS $(0=$ NOT VIBRATING; 1 = VIBRATING)

\begin{tabular}{|c|c|c|c|}
\hline Scenarios & Conditions & $\begin{array}{c}\text { Vibration } \\
\text { motor 1 }\end{array}$ & $\begin{array}{c}\text { Vibration } \\
\text { motor 2 }\end{array}$ \\
\hline \multirow{2}{*}{$\begin{array}{c}\text { 1 } \\
(\mathrm{D}=\text { obstacle } \\
\text { distance detected })\end{array}$} & $\mathrm{D}<80 \mathrm{~cm}$ & 0 & 0 \\
\cline { 2 - 4 } & $120 \mathrm{~cm}<\mathrm{D}<150 \mathrm{~cm}$ & 1 & 1 \\
\hline \multirow{2}{*}{$\begin{array}{c}2 \text { and } 3 \\
(\mathrm{D}=\text { distance from } \\
\text { foot to the ground })\end{array}$} & $2 \mathrm{~cm}<\mathrm{D}<120 \mathrm{~cm}$ & 1 & 0 \\
\cline { 2 - 4 } & $2 \mathrm{~cm}<\mathrm{D}<10 \mathrm{~cm}$ & 1 & 1 \\
\cline { 2 - 4 } & $\mathrm{D}<2 \mathrm{~cm}$ & 0 & 0 \\
\hline
\end{tabular}

\section{Experiments}

Both the wired and wireless configurations of the proposed device were tested in a laboratory environment for functionality validation. However, only the wired communication has been successfully set up for user trials. Discussions on a few observations are presented as follows.

With regards to the data communication between the two units, the wired configuration of the proposed device demonstrated good reliability when being tested. However, the wired connections between the sensor unit and the feedback unit tend to restrict the locations that the feedback unit can be installed on the user. On the other hand, the wireless configuration of the device was found to be unreliable at times. Occasionally, the feedback unit stopped responding to signals sent from the sensor unit, causing mistaken vibration signals to be sent to the user. However, the wireless configuration of the device would remove the hindrance caused by the connecting wires from the wired version, and hence the user has greater flexibility in the placement of the feedback unit. In addition, the RF modules require an additional power supply to support effective wireless data transmission over a certain distance and this means more space is needed in the platform to contain the additional battery.

Regarding the applicability of the proximity sensors and the microcontrollers, it was noted during the experiments that the use of the analog distance sensor for obstacle detection has certain limitations. Based on the experiments, it was noticed that the microcontroller is not able to generate precise signals. This lack of precision in signal generation between the microcontroller and the analog distance sensor risk sending false signals to the user and thus heavily reduces the performance of the device. On the other hand, the medium range sensor provides a more reliable TTL signals that can be directly processed by the microcontroller. The sensor simply gives a low signal when an obstacle is within range and a high signal when an obstacle is out of range. Compared to the analog distance sensor, the TTL signals from the medium range sensor tend to provide more reliable feedback signals to the user and do not generate any stray signals that may give rise to weak vibration signals, which can be misleading.

\section{CONCLUSION}

This research has investigated the application of IR sensors, microcontroller systems and wireless communication technologies for the development of an AT device that provides user-friendliness to visually-impaired users by providing hand-free hardware configuration. Such a device demonstrates the feasibility to be used by visually impaired to supplement their mobile navigation using a white cane. Worn under the shoes by the visually impaired users, this shoemounted device will help them detect obstacles and surface reflectance while constant feedback is provided to the users with a greater sense of their surroundings and instill confidence. Applying the surface reflectance detection method to identify the stance phase of the walking cycle is one novelty of this device. This device is a more cost-effective solution than other shoe-mounted devices using inertial sensors or computer vision technologies. The device can be used for leg rehabilitation purposes by sending a notification signal to the user if he fails to keep his feet above a certain level.

Two configurations of the proposed application have been built and validated to identify the problems and limitations. Improvements and potentials can be made. Firstly, with the concept of embedding electronics in a platform to be worn under the shoe of the users, more applications can be developed by incorporating other sensors within the platform. Pressure sensor, flex sensor and accelerometer are some of the sensors that can be used to monitor muscle and foot movements of the users. Secondly, the dimension and weight of the sensor unit and the feedback unit can both be further reduced through developing a printed circuit board for the device, and with smaller chips and batteries providing similar functionalities. Thirdly, temporal signal analysis can be considered to detect moving objects or living creatures in front of the user, e.g., human, animals, etc. If continuous signals with 
increasing magnitudes, i.e., decreasing distances, are measured, the system can determine the speed of the approaching object and compare it with the normal walking speed. In this way, the system can determine the object in front as a still object or an object moving fast towards the user. In the latter case, the user can be more prepared to the possible dangers in the dynamic environment than using only the white cane.

For the developed prototype, the research is focused on verifying the feasibility of the prototype using off-the-shelf components. The sensors and the chips are at the cheaper level in the market. With regard to the wireless configuration of the device, other types of higher cost wireless technologies, such Zigbee and Bluetooth, will be explored and used to replace the RF wireless modules to provide the required stability in wireless data transmission between the two units. However, production cost of any AT devices must be carefully considered as the target users are likely to command a low income due to their physical impairment. The device with wired data communication will be cheaper since fewer components are needed in it. The proof-of-concept prototype will be improved in both the functionalities and the mounting platform design so that direct contact with the ground can be avoided. User evaluation will be carried out to verify the functionalities and assess the potentials for commercialization.

\section{REFERENCES}

[1] M. A. Hersh and M. A. Johnson (editors), Assistive Technology for Visually Impaired and Blind People, London: Springer-Verlag, 2008.

[2] R. Stirling, K. Fyfe, and G. Lachapelle, "Evaluation of a new method of heading estimation for pedestrian dead reckoning using shoe mounted sensors," The Journal of Navigation, vol. 58, pp. 31-45, 2005.

[3] S. Brewster, "Visualization tools for blind people using multiple modalities," Disability and Rehabilitation, vol. 24, pp. 613-621, 2002.

[4] S. Ohtsuka, N. Sasaki, S. Hasegawa, and T. Harakawa, "Body-Braille system for disabled people", Proceedings of the 11th International Conference on Computers Helping People with Special Needs, Linz, Austria, pp. 682-685, July 9-11, 2008.

[5] F. Cecelja, V. Garaj, Z. Hunaiti, and W. Balachandran, "A navigation system for visually impaired," Proceedings of the 2006 IEEE Instrumentation and Measurement Technology Conference, Sorrento, Italy, pp. 1690-1693, April 24-27, 2006.

[6] L. Ran, S. Helal, and S. Moore, "Drishti: an integrated indoor/outdoor blind navigation system and service," Proceedings of the 2nd IEEE Annual Conference on Pervasive Computing and Communications, Orlando, Florida, USA, pp. 23-30, March 14-17, 2004.

[7] D. A. Ross and B. B. Blasch, "Development of a wearable computer orientation system," Personal and Ubiquitous Computing, vol. 6, pp. 4963, 2002.

[8] Y. Shiizu, Y. Hirahara, K. Yanashima, and K. Magatani, "The development of a white cane which navigates the visually impaired," Proceedings of the 29th Annual International Conference of the IEEE EMBS, Lyon, France, pp. 5005-5008, August 23-26, 2007.

[9] E. D'Atri, C. M. Medaglia, E. Panizzi, and A. D'Atri, "A system to aid blind people in the mobility: a usability test and its results," Proceedings of the 2nd International Conference on Systems, Sainte-Luce, Martinique, pp. 35, April 22-28, 2007.

[10] S. Koide and M. Kato, "3-D human navigation system with consideration of neighboring space information," Proceedings of the 2006 IEEE International Conference on Systems, Man, and Cybernetics, Taipei, Taiwan, pp. 1693-1698, October 8-11, 2006.

[11] J. S. Zelek, "Seeing by touch (haptics) for wayfinding," International Congress Series, vol. 1282, pp. 1108-1112, 2005.
[12] F. Maingreaud, E. E. Pissaloux, R. Velázquez, F. Gaunet, M. Hafez, and J-M. Alexandre, "A dynamic tactile map as a tool for space organization perception: application to the design of an electronic travel aid for visually impaired and blind people," Proceedings of the 2005 IEEE Engineering in Medicine and Biology 27th annual conference, Shanghai, China, pp. 6912-6915, September 1-4, 2005.

[13] G. Sainarayanan, R. Nagarajan, and S. Yaacob, "Fuzzy image processing scheme for autonomous navigation of human blind," Applied Soft Computing, vol. 7, pp. 257-264, 2007.

[14] M. Sarfraz and S. M. A. J. Rizvi, "Indoor navigation aid system for the visually impaired," Proceedings of the 2nd International Conference on Geometric Modeling and Imaging, Zurich, Switzerland, pp. 127-132, July 4-6, 2007.

[15] W. C. Lee and M. K. H. Leung, "SINVI: smart indoor navigation for the visually impaired," Proceedings of the 8th International Conference on Control, Automation, Robotics and Vision, Kunming, China, pp. 10721077, December 6-9, 2004.

[16] S. Kaluwahandi and Y. Tadokoro, "Portable travelling support system using image processing for the visually impaired," Proceedings of the 2001 International Conference on Image Processing; Thessaloniki, Greece, pp. 337-340, October 7-10,2001.

[17] G. Iannizzotto, C. Costanzo, P. Lanzafame, and F. L. Rosa, "Badge3D for visually impaired," Proceedings of IEEE Computer Society Conference on Computer Vision and Pattern recognition, San Diego, CA, USA, pp. 29, June 20-26, 2005.

[18] B. S. Tjan, P. J. Beckmann, R. Roy, N. Giudice, and G. E. Legge, "Digital sign system for indoor wayfinding for the visually impaired," Proceedings of the 2005 IEEE Computer Society Conference on Computer Vision and Pattern Recognition, San Diego, CA, USA, pp. 30 June 20-26, 2005.

[19] Y. Ebrahim, W. Abdelsalam, M. Ahmed, and S. C. Chau SC, "Proposing a hybrid tag-camera-based identification and navigation aid for the visually impaired," Proceedings of the 2nd IEEE Consumer Communications and Networking Conference, Nevada, USA, pp. 172177, January 3-6, 2005.

[20] Artificial vision for the totally blind, available from: http://www.artificialvision.com/ (Last accessed: Oct 14, 2009).

[21] L. A. Johnson and C. M. Higgins, "A navigation aid for the blind using tactile-visual sensory substitution," Proceedings of the 28th IEEE EMBS Annual International Conference, New York, USA, pp. 6289-6292, August 29-September 3, 2006.

[22] E. Foxlin, "Pedestrian tracking with shoe-mounted inertial sensor," IEEE Computer Graphics and Applications, vol. 25, pp. 38-46, 2005.

[23] S. Godha and G. Lachapelle, "Foot mounted inertial system for pedestrian navigation," Measurement Science and Technology, vol. 19, 075202, 2008.

[24] M. Chen, B. Huang, and Y. Xu, "Intelligent Shoes for Abnormal Gait Detection," Proceedings of the 2008 IEEE International Conference on Robotics and Automation, Pasadena, CA, USA, pp. 2019-2024, May 19-23, 2008 .

[25] K. Kong and M. Tomizuka, "A gait monitoring system based on air pressure sensors embedded in a shoe," IEEE/ASME Transactions on Mechatronics, vol. 14, pp. 358-370, 2009.

[26] T. Liu, Y. Inoue, and K. Shibata, "Development of a wearable sensor system for quantitative gait analysis," Measurement, vol. 42, pp. 978 988,2009

[27] P. Fitzpatrick and C. C. Kemp, "Shoes as a platform for vision," Proceedings of the 7th IEEE International Symposium on Wearable Computers, New York, USA, pp. 231-234, October 21-23, 2003.

[28] S. Barrera, P. Romanos, S. Saito, H. Takahashi, and M. Nakajima, "WARAJI: foot-driven navigation interfaces for virtual reality applications", In: K. Aizawa, Y. Nakamura, and S. Satoh (editors), Advances in Multimedia Information Processing - PCM 2004, pp. 1-7, Berlin Heidelberg: Springer-Verlag, 2004.

[29] B. Rey, J. A. Lozano, M. Alcañiz, L. Gamberini, M. Calvet, D. Kerrigan, and F. Martino, "Super-feet: a wireless hand-free navigation system for virtual environments," In: R. Shumaker (editor), Virtual Reality, pp. 348-357, Heidelberg: Springer Berlin, 2007. 